\title{
Multi-objective design of single room ventilation units with heat and water recovery
}

\author{
Antoine Parthoens ${ }^{* 1}$, Luc Prieels ${ }^{2}$, Jean-Jacques Embrechts ${ }^{3}$, Yves Detandt ${ }^{4}$, \\ Sébastien Pecceu ${ }^{5}$, Samuel Gendebien ${ }^{1}$ and $\underline{\text { Vincent Lemort }}^{1}$ \\ 1 Thermodynamics Laboratory, ULiège \\ Allée de la découverte 17 \\ 4000 Liège, Belgium \\ *Corresponding author: a.parthoens@uliege.be \\ 2 Greencom SPRL \\ Rue Gilles Magnée 92/3 \\ 4430 Ans, Belgium \\ 3 IntelSig, ULiège \\ Allée de la découverte 10 \\ 4000 Liège, Belgium \\ 4 FFT \\ Rue Emile Francqui 9 \\ 1435 Mont-Saint-Guibert, Belgium \\ 5 Belgian Building Research Institute \\ Avenue P. Holoffe 21 \\ 1342 Limelette, Belgium
}

\begin{abstract}
The present paper describes the design improvement of a single-room ventilation unit. This ventilation system presents many advantages, however, several drawbacks exist. The first one is the acoustic disturbance. As the facilities are directly installed within the rooms, the fans' noise may create discomfort. Furthermore, in the cold or temperate climates, condensation or frost may appear. A dedicated management should then be implemented. Finally, as the system is not centralized, communication between the different units is required to ensure the global system efficiency. A team of several industrial partners and research institutes tackles the above-mentioned issues in the frame of the "Silenthalpic" project. The project is split in three major tasks. To correctly reduce the sound emission level (i), a spectral analysis of the noise emitted by an existing unit was undertaken, revealing that frequencies under $1 \mathrm{kHz}$ are mainly responsible for the noise disturbance. From this analysis, active and passive solutions for noise reduction are envisaged, showing encouraging trends. The next research aspect is the exchanger of the ventilation unit (ii). The constituting material is a new porous membrane allowing the humidity transfer (vapor or liquid). This specific exchanger is numerically modelled to predict its performances. The last considered problematic is the optimization of the ventilation and control strategies for the specific case of decentralized units (iii), taking advantage of sensors and recent communication technologies like IOT (Internet Of Things) to establish communication between decentralized units and ensure their consistent control. The association of the three aspects presented here should then lead to versatile and efficient ventilation systems.
\end{abstract}

KEYWORDS

Single-room ventilation systems, noise reduction, heat recovery, humidity recovery,

\section{INTRODUCTION}

\subsection{Global context}

In the context of global warming, energy savings in buildings are getting more and more important. Eurostat (Eurostat, 2018) stands that in European Union in 2016, the households accounted for $25.4 \%$ of the final energy consumption. That is the reason why new and refurbished buildings are getting more isolated and airtight. This leads to an increase of the energy consumption relative proportion dedicated to ventilation (Roulet, Heidt, Foradini, \& 
Pibiri, 2001). To reduce this aforementioned consumption, mechanical ventilation with heat recovery was introduced. The most widespread system is a centralized system with a single heat exchanger and a ducting networks. Besides this technology, decentralized systems, also known as single-room ventilation with heat recovery systems (SRVHR) do exist. The purpose of the present paper is to describe a project gathering multiple research centres and industrials aiming at developing a new efficient decentralized ventilation unit.

\subsection{Issues of decentralized ventilation systems and challenges set in the Silenthalpic project}

SRVHR are units composed of a casing containing one or two fans, a heat exchanger, filters and control electronics. The whole setup is installed at the top of a window (thinnest part of a building), or simply against a wall with two drilled holes. A schematic representation of the unit is shown in Figure 1.

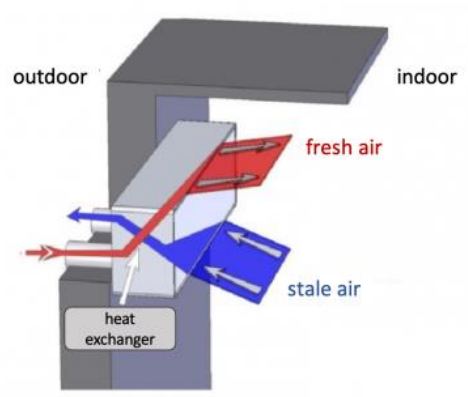

Figure 1 Schematic representation of SRVHR units

For a given building, the number of required units depends on the flow rates delivered by the considered system and the needs of the building, function of its surface, population and type of activity.

This technology already exists and presents some advantages such as :

- the installation easiness, especially in the frame of refurbishment;

- a reduced ducting network that leads to an easy system maintenance;

- a flexible implementation, as the number and the position of the units are modular;

- a pre calibrated mass flow rate.

The objective of the "Silenthalpic" project is to develop a compact unit, limiting three major issues:

- Noise disturbance limitation. As the solution is decentralized, fans are in the actual living rooms (bedrooms, offices,...) and the noise generated must be limited. Specific efforts are deployed to maintain the acoustic disturbance linked to the use of fans as low as possible. Moreover, the ventilation unit is prone to increase the transmission of exterior noise emissions to the building's interior. Acoustic insulation properties if the unit should be evaluated.

- Enthalpy exchanger development. In addition to recover heat, it also recovers a part of the moisture from the extracted stale air.

- Development of a so-called "evolutionary" ventilation system.

During the development of all latter ones, the need of compactness of the system is always kept in mind. 


\subsection{Methodology}

The first research aspect focuses on the aero-acoustic phenomena related to the mechanical ventilation. After a first stage of advanced diagnostic on an off-the-shelf unit, modelling methods are developed to predict the involved phenomena. Another point is the investigation of the application of the active and passive noise reduction to ventilation units. The target is to reach a standard sound level of $35 \mathrm{dBA}$ for a flowrate of $50 \mathrm{~m}^{3} / \mathrm{h}$.

The second research axis deals specifically with the possibilities of heat and humidity transfer within so-called "enthalpy" membranes. The target is to develop membrane able to recover 50\% of the recoverable water quantity. This should reduce condensate issues and delays frost-related problems, while evacuating building excess moisture. In tertiary applications, as well as in dry buildings and colder climates, this increases occupant comfort and provides an important technological advantage. To achieve this, the project focuses on the development of an enthalpy membrane. This membrane will then be used to manufacture a heat exchanger core, later tested and integrated into ventilation units.

Finally, the project focuses on aspects related to the overall integration of ventilation systems. The ventilation strategies made possible by this new technology are studied in the light of the regulatory frameworks of the various surrounding countries. These strategies are evaluated and modelled. They take into account the new potential offered by the Internet of Things (IOT) for users as well as for manufacturers, installers and home automation activities. All the results of this work will then be implemented into two applications: 2 window and 2 lintel demonstrators, which will take into account the results of the LCA (Life Cycle Assessment) studies. Finally, the final system will be tested in different acoustic and thermal test cells to estimate improvements.

\section{METHODS FOR THE REDUCTION OF NOISE EMISSIONS BY THE VENTILATION UNIT}

Several tasks have been defined in order to reduce the noise generated by the ventilation unit in the interior living area. Passive and active solutions installed on the unit itself are envisaged to attenuate the noise emissions by the two fans.

\subsection{Numerical model of the ventilation unit}

The acoustic improvements of the design require a deep understanding of the noise sources characteristics and the acoustic transmission properties of the different components of the ventilation unit. Acoustic simulations provide access to the whole pressure field (spatial and frequency dependence) which leads to a better understanding of the propagation mechanisms and the dominant contributions perceived.

Although acoustics deal with unsteady pressure fluctuations, the flow solvers are designed to accurately represent the turbulent fields and their schemes are not tailored to preserve at the same time the dissipation and dispersion properties of the acoustic waves. Based on the unsteady flow solution, the acoustic solver Actran computes the aeroacoustic noise sources following the original idea of Lighthill 1952. The acoustic propagation is modelled by a finite element discretization of the acoustic domain. The mesh is designed to accurately propagate the acoustic waves up to the highest frequency. The solver works in the frequency domain, computing each frequency separately and updating the material properties according to the characteristics defined by the user. 

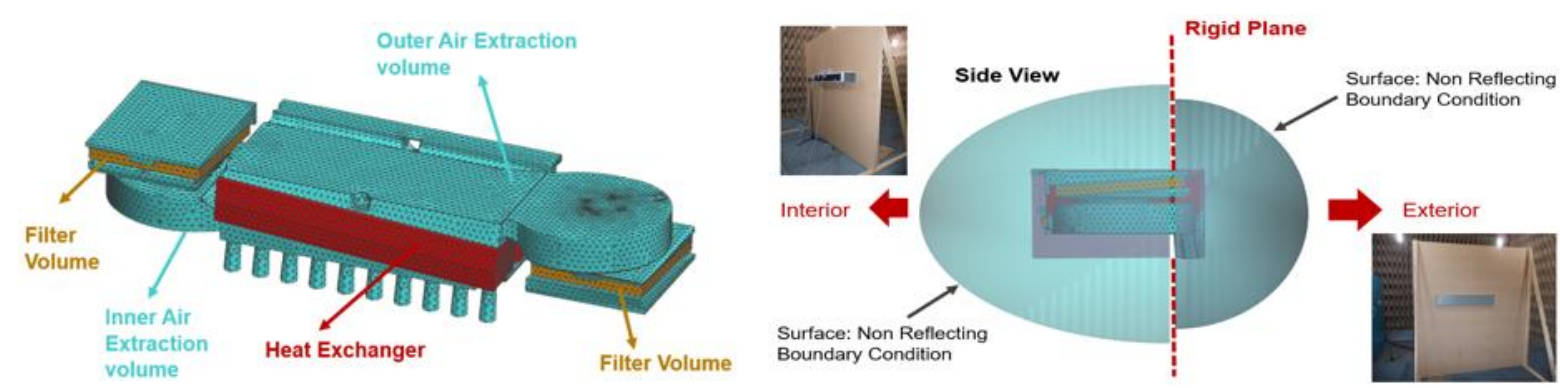

Figure 2 Acoustic model of fan and heat exchanger unit (left) and global view of the acoustic model (right)

In the current project, the main simulation challenge was related to the modelling of the heat exchanger part. Discretizing the small channels is not practical as it would lead to very expensive simulations due to the large number of elements. The alternative modelling developed in this research project was to consider the exchanger as a whole system connecting the inlet to outlet with an equivalent fluid mixing in between. The viscous and thermal effects affecting the acoustic propagation in the system are included in the model. Most of the parameters are directly related to geometrical and mass flow rate properties and a very small set is more complex as related to the acoustic transfer properties between the channel pipes. These parameters have been set based on measurements in the Acoustic Laboratory of the University of Liège. The heat exchanger model has been validated in the framework of the Silenthalpic project and could be extended to any heat exchanger, while reducing significantly the cost compared to a simulation based on all channels discretized.

The acoustic model has been used to analyse the transfer properties and different acoustic modes existing in the system. As the fan spinning generate a series of tonal components, the rotation speed should be controlled to avoid a match between the blade passing frequencies emitted by the fan and the natural resonant frequencies of the system.
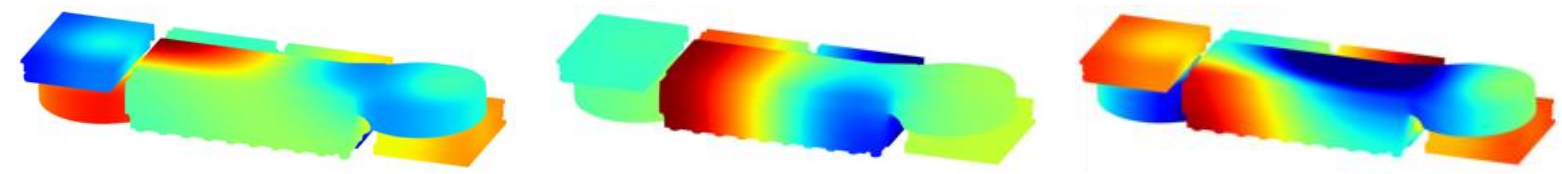

Figure 3 Acoustic pressure modes of the system at $428 \mathrm{~Hz}$ (left), $664 \mathrm{~Hz}$ (middle) and $714 \mathrm{~Hz}$ (right)

\subsection{Experimental diagnosis of the ventilation unit}

A vibro-acoustic evaluation of the ventilation unit in its initial state was first carried out. This was used as a starting point to quantify the further improvements brought by the passive as well as the active solutions to be developed by the different research teams. The acoustic insulation of the ventilation unit inserted into a hard wall has been measured in a reverberant room, according to the standard EN ISO 10140-2. With the same set-up, the sound pressure levels emitted by the two fans were measured according to EN ISO 100052:2005. The standard sound level measured in the room at $50 \mathrm{~m}^{3} / \mathrm{h}$ was $41.6 \mathrm{~dB}(\mathrm{~A})$, with the most part of the energy emitted between 200 and $1000 \mathrm{~Hz}$. An objective of the research project is to reduce this level to 35 $\mathrm{dB}(\mathrm{A})$. The test in the reverberant room has also shown a significant influence of the anti-dust filters installed above each fan.

In parallel with these measurements, another set-up was realized in an anechoic room with a similar ventilation unit which was inserted into a big baffle. This set-up has been used to measure the directivity of the sound emission by each fan, in several third-octave bands. 
Globally, the emission was shown to be close to omnidirectional, for the frequency interval of interest. Also in the anechoic room, high-resolution spectra $(\Delta \mathrm{f}=1 \mathrm{~Hz})$ were measured at several positions. Pure tones were of course detected in the spectra and their frequency was related to the rotation speed of the fans and their number of blades.

Finally, intensimetry and an acoustic camera were used to identify the main sources of noise emission on the interior side of the module. Both fans contribute significantly to the noise emissions, in particular at the air inlet aperture.

\subsection{Passive attenuation of the noise emissions}

Several methods of passive acoustic attenuation are available, like the application of absorbing materials (mineral fibres, foams, ...) inside the ventilation unit, at well-defined locations. Dampers and quarter-wave resonators could also be used, if possible.

The experimental diagnosis has shown the predominance of the noise emitted through the air flow apertures. Therefore, several solutions have been tested during the first part of the research:

- a perforated absorbing front cover,

- different mufflers with $3 \mathrm{~cm}$ mineral wool applied in front of the air apertures,

- a muffler with $3 \mathrm{~cm}$ mineral wool inserted into the front cover.

The best results were obtained with the second solution, with a noise reduction of $7 \mathrm{~dB}(\mathrm{~A})$. However, this solution cannot be implemented in practice, since it significantly increases the global volume of the ventilation unit. Also, the influence of the mufflers on the air flow (in $\mathrm{m}^{3} / \mathrm{h}$ ) has not been investigated yet. However, this first result has confirmed the predominance of the noise emitted through the air inlet aperture: if this noise source can be properly attenuated, then the objective of a maximum noise level of $35 \mathrm{~dB}(\mathrm{~A})$ at $50 \mathrm{~m}^{3} / \mathrm{h}$ is achievable.

The numerical model of the ventilation unit described in section 2.1 will also be used to test other passive solutions. This kind of model can be particularly useful to optimize some parameters such as the type, the location or the thickness of a layer of absorbing material.

\subsection{Active attenuation of the noise emissions}

Active noise control (ANC) is a method of sound attenuation in which a secondary acoustic wave is generated by an actuator (generally a loudspeaker) to superpose the primary (noise) wave, in such a way that the superposition of both waves results in a reduced amplitude. In theory, if the secondary wave is such that the opposite pressure signal is generated in the immission zone, then a perfect silence is obtained. In practice, the extension of the zone in which attenuation is observed is limited and the noise reduction itself is limited, according to the method used for the control and the quality of the material.

The analysis of the scientific and technical literature has revealed some interesting studies and practical realizations (mostly in laboratory) for the ANC of ventilation units. In particular, methods using the control of fan noise propagating into a duct have shown good results. Also the control of noise propagating through an 'open window' has recently attracted the interest of the ANC community, especially to reduce the noise emissions of relatively small equipment like the ventilation unit.

The experimental diagnosis has shown the predominance of the noise emitted through the air flow apertures, in particular the air inlet aperture on the interior side of the building. It was therefore decided to first design and study the solution of an additional duct, fixed to the existing ventilation module and deporting the air inlet aperture to the end of the duct, approximately $1 \mathrm{~m}$ 
away of its initial position. This solution allows the ANC of the noise emitted by the fan extracting air through this aperture, while still maintaining the air flow.

ANC in a duct is usually realized with a feedforward control system. This system consists of a sensing microphone measuring the noise upstream of the controller, the controller itself (a digital signal processor DSP), one or several loudspeakers creating the secondary wave in the duct and one or several error microphones downstream to measure the effect of the control. The length of the duct is chosen to guarantee a sufficient delay of sound propagation between the sensing microphone and the loudspeakers, such that the DSP would have enough time to generate the secondary wave. Some systems don't need this sensing or reference microphone: the controller only needs the error microphones' signals as input data (feedback ANC). However, the stability is better in feedforward systems, the main reason why this solution has been selected in this research.

The duct has been built with a rectangular cross-section, the dimensions of which have been selected such that only plane waves are allowed to propagate up to about $1200 \mathrm{~Hz}$ (this allows to use only one loudspeaker and one error microphone). ANC indeed usually shows good performance below $1 \mathrm{kHz}$ (even below $500 \mathrm{~Hz}$ sometimes) which is considered as the frequency interval of interest. For higher frequencies, a combination with passive acoustic absorbers is usually envisaged, which will be the case here.

Finally, strategic locations must be defined for the two microphones and the loudspeaker. For this purpose, the numerical model described in section 2.1 will be used to tests several combinations of microphones and loudspeaker's positions and select the one(s) reaching the greatest noise reduction.

\section{DEVELOPMENT OF HEAT AND MOISUTRE RECOVERY EXCHANGER}

Current enthalpy recovery systems imply devices such as rotating wheels (Zeng, Liu, \& Ashish, 2017) that are active or imply exchangers made of fragile porous materials such as paper. The off-the-shelf ones are either complicated to implement or present a limited life-span. The Silenthalpic proposal is to develop a robust enthalpy exchanger with a high enlargement factor (i.e. ratio between developed and flat surfaces) allowing an efficient heat, vapor and liquid water transfer.

The development of the membrane itself at the lab and industrial scales and the development of a numerical model predicting the performance of the exchanger are tackled. The aim of the numerical model is to perform an optimization of the exchanger design.

\subsection{Porous membrane development}

The main objective here is to develop a composite membrane allowing water to transfer from one of its side to the other and that can be thermoformed.

The chosen method was to inject some solid particles into a polymer matrix to artificially create some defects. Those latter ones must have a characteristic size allowing the transfer of water particles by capillarity while blocking the transfer of air. Particles of different sizes are tested to finally select the one fitting the best the application.

The first step of the process is extruding the mixture. The polymer materials as well as the solid particles are being dried several hours to obtain a $-40^{\circ} \mathrm{C}$ dewpoint. Then the mix is extruded in a bivis co-rotative extruder. The solid particles powder is added by a side-feeder leading to an easily obtained homogenous grains. From this product, films must be manufactured. The 
previous product is dried again to reach humidity lower than 200 PPM. Then, films are made with a single vis extruder. These composite films can then be thermoformed to get a high enlargement factor as well as the desired shape, depending on the exchanger design.

To evaluate the ability of the membrane to transfer water and especially liquid water, an experimental apparatus has been put in place.

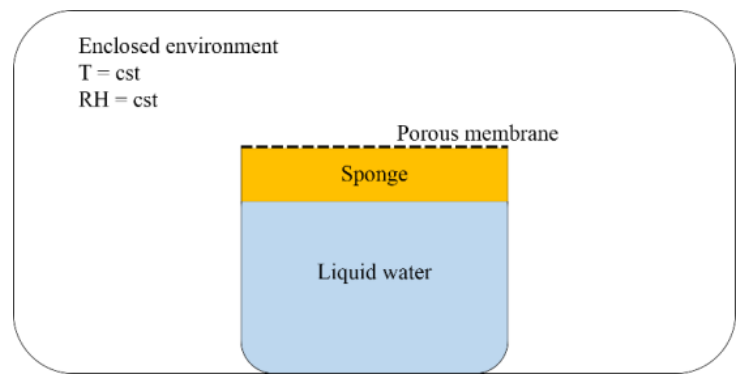

Figure 4 Experimental setup used for the membrane permeability determination

Preliminarily, the membrane is submerged in water for one hour to load all the interstices and to measure steady state conditions. Then, a recipient is filled with liquid water and closed with the membrane. To ensure a permanent contact between the liquid and the membrane, a sponge is added and periodic checks were made to control that the liquid was in contact with the sponge during the whole experiment. This setup is weighted and put in an enclosed environment where the atmospheric conditions were controlled. The temperature is fixed to $23 \pm 0.5^{\circ} \mathrm{C}$ and the relative humidity to $50 \pm 1 \%$. After 24 hours in these conditions, the recipient is weighted a second time. The difference between the two measures corresponded to the water that went through the membrane and that was evaporated to the environment.

\subsection{Numerical model implementation}

In order to get a suitable enthalpy exchanger, a design process is needed. The objective is to exchange the largest amount of heat, the optimal quantity of water, to limit the pressure drops and to keep the compactness.

Many works in scientific literature handle enthalpy exchangers. Dugaria et al (Dugaria, Moro, \& Del Col, 2015) predicted with a 2-dimensional finite difference model sensible, latent and total effectiveness sensitivity to membranes' characteristics. Sebai et al. (Sebai, Chouikh, \& Guizani, 2014) modelled cross-flow enthalpy exchangers with balanced or unbalanced flow with a control volume method. Zhang (Zhang, 2009) measured latent effectiveness going up to about $70 \%$ with a paper-plate and paper-fin exchanger. Niu \& Zhang (Niu \& Zhang, 2001) showed numerically and experimentally the evolution of enthalpy exchangers for different membranes and operating conditions.

However, all the above-mentioned works only consider a vapour transfer. In the frame of this project, a special attention is paid to the liquid water transfer for the condensates management. A specific model has been developed to handle this kind of mass transfer coupled with a heat transfer. This model is detailed by Parthoens et al. (Parthoens, Gendebien, Lemaitre, \& Lemort, 2018). All in all, it allows to predict the sensible, latent and total efficiencies for given atmospheric conditions (indoor and outdoor), mass flow rates and for a given geometry and a membrane permeability.

The final objective will be to supply different geometries with specific constraints to the model and to assess which one will be produced and implemented in the actual demonstrator. 


\section{VENTILATION SYSTEM DESIGN AND CONTROL AT BUILDING SCALE 4.1 Design and control strategies with decentralized ventilation}

Despite the advantages of decentralized ventilation (see $\S 1.1$ ), one of its main disadvantages is that the total air change rate at building scale may be twice as high as with a conventional mechanical system. Indeed, air is supplied and exhausted in every space with a decentralized unit, while with a classical system air is generally supplied in "dry" spaces (living, bedrooms, etc) and exhausted in wet spaces (kitchens, bathrooms, etc). The same fresh air is thus used for two spaces with classical systems.

This drawback can however be tackled in two ways:

- Using demand controlled ventilation (DCV). The ventilation needs in dry and wet spaces are not always synchronous, and the decentralized approach allows a differentiated control per space. Even if the installed flow capacity (total design flow) is higher than for conventional system, the relative flow rate reduction due to DCV is probably much higher with decentralized systems.

- The developed unit has been designed so that is possible do connect a duct at the extraction side, allowing displacing the exhaust a few meters from the ventilation unit itself. This 'remote extraction' configuration is straightforward in a two-room accommodation (bedroom/bathroom) like hotels or rest houses. This 'remote extraction' principle could also be applied in small houses by grouping dry and wet rooms by two. This way of working is closer to conventional system, but would still require much less ducting (shorter, narrower) than a centralized installation. The main advantages of decentralized systems would thus persist.

Multizone simulations were performed with the CONTAM software to evaluate the impact of decentralized ventilation on the overall energy performance, and showing how the above solutions help in reducing its energy footprint. One of the test cases is the one of a two bedrooms apartment as illustrated at Figure 5. The design flows were chosen supposing 3 occupants and a nominal flow of $25 \mathrm{~m}^{3} / \mathrm{h} /$ pers (prEN 16798-1). Flow rates in wet spaces were chosen according current Belgian standards (NBN D-50-001) and/or to reach the balance at building level.
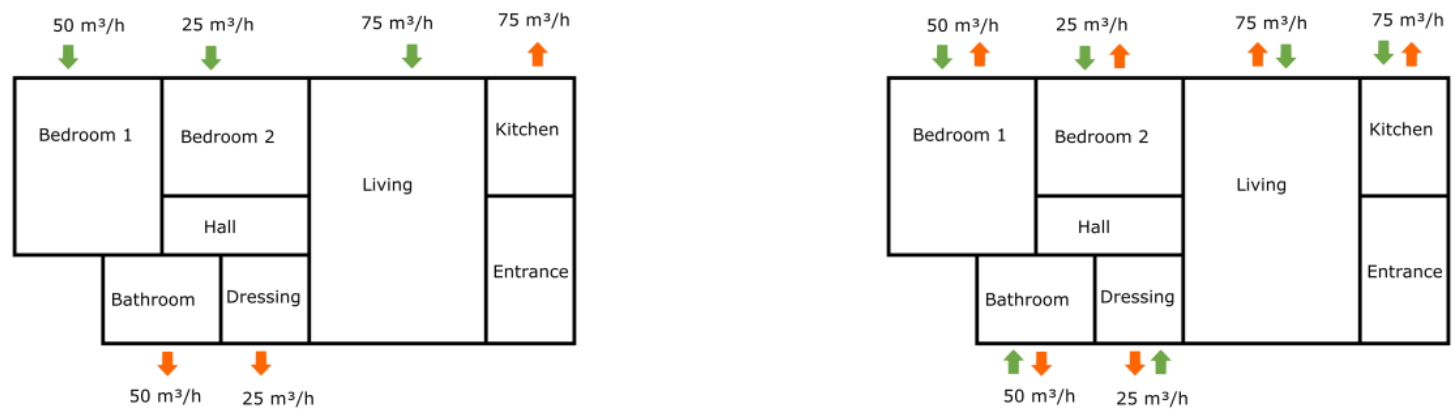

Figure 5 Example apartment and flow rates for a conventional mechanical system (left) and a fully decentralized system (right). With these flow rates, the total flow rate for the conventional system is $150 \mathrm{~m}^{3} / \mathrm{h}$ while it is 300 $\mathrm{m}^{3} / \mathrm{h}$ for the fully decentralized system.

With the chosen flows, the 'remote extraction' solution could be easily applied to the 3 following pairs of dry and wet spaces:

- Bedroom 1 and Bathroom 
- Bedroom 2 and Dressing

- Living and Kitchen

In this precise configuration, this remote extraction solution has exactly the same nominal flow rate as a conventional mechanical system.

Demand controlled ventilation is simulated with a local detection and regulation in each space:

- Supply in dry spaces (or extraction in dressing): linear variation from $10 \%$ to $100 \%$ of nominal flow for CO2 concentration from 400 to $1000 \mathrm{ppm}$.

- Exhaust in wet spaces: linear variation from $10 \%$ to $100 \%$ of nominal flow for Relative humidity from $30 \%$ to $70 \%$.

Three types of "physical system" are simulated:

- The conventional system (centralized). The ventilation needs were computed per space, and the overall flow balance was ensured at any time (lower flow adjusted to reach the highest flow). The fully decentralized system as on the right side of figure 3 . In this case, the balance in only per space

- The 'remote extraction' system with the pairing proposed above. The nominal flows correspond to the left side of Figure 3, but the flow rates are balanced for each pair of rooms (corresponding to each physical ventilation unit).

Regarding pollutant sources, humidity sources are defined in the bathroom and kitchen at representative schedules, and the occupancy profile corresponds to two working adults with one child. Simulation results are expressed as mean ventilation flow rate (representative of the ventilation heat loss) over the simulation period and given in Table 1.

Table 1 Mean flow rate of the DCV strategy for different ventilation designs. The mean flow for the fully decentralized system remains higher than for the conventional system, but the relative reduction is much stronger. For this example, one also sees that the 'remote extraction' variant is nearly identical to the conventional system in terms of flow rate

\begin{tabular}{|c|c|c|}
\hline Case & Nominal flow rate $\left[\mathrm{m}^{3} / \mathrm{h}\right]$ & $\begin{array}{c}\text { Mean flow rate with local } \\
\text { regulation and detection } \\
{\left[\mathrm{m}^{3} / \mathrm{h}\right]}\end{array}$ \\
\hline $\begin{array}{c}\text { Conventional centralized } \\
\text { system }\end{array}$ & 150 & 63 \\
\hline Fully decentralized system & 300 & 86 \\
\hline $\begin{array}{c}\text { Remote extraction } \\
\text { decentralized system }\end{array}$ & 150 & 66 \\
\hline
\end{tabular}

On this example, results of Table 1 confirm the efficiency of the two envisaged strategies to reduce the mean flow rate of decentralized systems and the associated heat loss.

\subsection{Regulation of decentralized systems and communication between units}

The presented case in the above subsection is quite simple. In some configurations, several decentralized units could be needed to reach the required flow rates in some spaces (e.g. in living spaces). In that case, not all units will be equipped with sensors and their control should be coordinated.

An electronic card has been designed and prototyped with the ability to support all types of required sensors (temperature, pressure, $\mathrm{CO} 2$, etc) and to communicate with other units through the Zigbee protocol. This allows the different units in the same building to communicate 
between them (for a coordinated control) and to communicate to the outside for monitoring or diagnostic purpose.

\section{CONCLUSIONS}

The aim of the present paper was to present the work undertaken by a consortium of many industrials and research centres. The objective was to develop a highly efficient single room ventilation system with heat and humidity recovery. It was seen that three major axes were investigated.

After a deep analysis of existing systems, numerical and experimental investigations were carried on, to passively and actively reduce the noise emissions of the unit. Simultaneously, a new composite membrane was developed to built-up a robust and performing enthalpy exchanger. Finally studies have been driven to integrate intelligently such units at the building scale, respecting needs and standards.

\section{ACKNOWLEDGEMENTS}

The consortium would like to thank Walloon Region and the Pole Mecatech [convention 7711] for the support in this work.

\section{REFERENCES}

Dugaria, S., Moro, L., \& Del Col, D. (2015). Modelling heat and mass transfer in a membrane-based air-to-air enthalpy exchanger. 33rdUIT(Italian Union of Thermofluid-dynamics) Heat transfer conference.

Eurostat. (2018). Consumption of energy. Obtido em June de 2018, de http://ec.europa.eu/eurostat/statistics- explained/index.php?title=Consumption _of_energy\#End- users

Niu, M., \& Zhang, L. (2001). Membrane-based Enthalpy Exchanger: material considerations and clarification of moisture resistance. Journal of Membrane Science, 179-191.

Parthoens, A., Gendebien, S., Lemaitre, P., \& Lemort, V. (2018). Model development of a new enthalpy exchanger under wet conditions in the frame of a single room ventilation . System Simulation in Buildings. Liège.

Roulet, C.-A., Heidt, F., Foradini, F., \& Pibiri, M.-C. (2001). Real heat recovery with air handling units. Energy and Buildings, 33(5), 495-502.

Sebai, R., Chouikh, R., \& Guizani, A. (2014). Cross-flow membrane-based enthalpy exchanger balanced and unbalanced flow. Energy Conversion and Management, 1928.

Zeng, C., Liu, S., \& Ashish, S. (2017). A review on the air-to-air heat and mass exchanger technologies forbuilding applications. Renewable and Sustainable Energy Reviews, 75, 753-774.

Zhang, L.-Z. (2009). Heat and mass transfer in plate-fin enthalpy exchangers with different plate and fin materials. International Journal of Heat and Mass Transfer, 2704-2713. 